\title{
Tunnelling and the Born-Oppenheimer approximation in optical lattices
}

\section{S K Dutta and G Raithel}

University of Michigan, Physics Department, Ann Arbor, MI 48109-1120, USA

Received 19 January 2000

\begin{abstract}
We study periodic well-to-well flopping of rubidium atoms in one-dimensional grey optical lattices using a nondestructive, real-time measurement technique and quantum Monte Carlo wavefunction simulations. The observed flopping rates as well as flopping rates extracted from exact band structure calculations can largely be reproduced using adiabatic models that employ the Born-Oppenheimer approximation. The adiabatic model is greatly improved by taking into account a gauge potential that is added to the usual adiabatic light-shift potential. The validity of the adiabatic model allows us to interpret the observed flopping phenomenon as periodic well-to-well tunnelling. At low intensities and in related far-off-resonant optical lattices the adiabatic model fails. There, a weak-coupling model becomes valid, which describes the well-to-well flopping as a Rabi oscillation between weakly coupled states, but not as a tunnel effect.
\end{abstract}

Keywords: Optical lattices, Born-Oppenheimer approximation, gauge potential, tunnel effect

\section{Introduction}

Optical lattices are periodic light-shift potentials of atoms, created by the interference of multiple laser beams. Atoms can be laser-cooled and localized in these potentials. The rate $\gamma$ at which the trapped atoms scatter lattice photons is an essential parameter for both the atomic temperature and the lifetime of coherent atomic centre-of-mass (CM) motion. A low value of $\gamma$ generally leads to lower temperatures, larger vibrational ground state populations, and long coherence lifetimes. A large variety of optical lattices has been realized [1], including lattices in which $\gamma$ is minimized using high-intensity, far-off-resonance laser light (FORL) [2] and/or using laser light that is blue-detuned with respect to the relevant atomic transition (grey lattices [3]). CM wavepackets of atoms in optical lattices have been produced, and their properties and decay mechanisms have been studied [4]. Using low-intensity grey lattices and FORLs, the coherence decay rate could be further reduced to a level where quantum tunnelling [5-7] and Bloch oscillations [8] became visible. In this regime, the atoms migrate through the lattice via well-to-well or longer-range quantum transport, and not via quasi-classical diffusion induced by excitations into free lattice bands and subsequent recapture into lattice wells [9]. These two migration mechanisms are fundamentally different: the quasi-classical diffusion is not based on coherent quantum motion, and its rate increases with $\gamma$. In contrast, quantum transport is based on coherent, quantized CM dynamics, and its significance decreases with increasing $\gamma$.
As a result of the spinor nature of the atomic wavefunction, one can distinguish between optical lattices for which the Born-Oppenheimer approximation (BOA) applies, and others for which the BOA fails $[10,11]$. If optical lattices differ with respect to the applicability of the BOA but have similar adiabatic potential surfaces, their quantum transport rates can be very different. Further in the case of adiabatic spin motion, i.e. in the regime in which the CM dynamics is slow enough to be adiabatically separable using the BOA, the quantum CM motion on the adiabatic potential surfaces can be significantly altered by topological effects [12]. One such effect, the influence of gauge potentials on the quantum transport in certain grey optical lattices, has recently been observed $[7,10]$. In contrast to the usual adiabatic potentials of the BOA, which are proportional to the lattice intensity, the gauge potentials solely depend on the geometry of the lattice, not on its intensity.

In this paper, we study well-to-well flopping-a form of quantum transport-in optical lattices formed by pairs of counterpropagating, linearly polarized laser beams with orthogonal (lin $\perp$ lin case) or arbitrary (lin $\theta$ lin case) angles between the polarization directions. In section 2 we discuss the BOA in optical lattices, and the conditions under which gauge potentials are significant. In our experiments, we use a nondestructive, real-time technique to measure periodic tunnelling currents, described in section 3. In section 4 we then review our previous experiments and simulations on the intensity-dependence of the well-to-well flopping frequency in lin $\perp$ lin grey optical lattices. We compare our previous results with the results of exact band structure 
calculations and band structure calculations that are based on the BOA. We use a weak-coupling model, which is explained in detail in section 6 , to understand the generic decrease of the flopping frequency observed at very low intensities. We then shift our attention to the $\theta$-dependence of the well-towell flopping behaviour in $\operatorname{lin} \theta$ lin grey lattices. There, the main signal we measure is generated by periodic tunnelling between fixed pairs of neighbouring wells. In section 5 we present measurements and wavefunction simulations of the $\theta$-dependence of the tunnelling frequency, as well as corresponding results obtained with adiabatic and weakcoupling models. In sections 4 and 5 we largely find validity of the BOA. In section 6 we apply our adiabatic and weakcoupling models to the well-to-well flopping in a FORL that displays periodic double-well potentials that appear similar to the ones found in $\operatorname{lin} \theta$ lin grey optical lattices [11]. Section 7 concludes the paper with a discussion of the main results.

\section{The Born-Oppenheimer approximation in optical lattices}

The position-dependent atom-field coupling in optical lattices can be written as a spatially periodic effective potential operator $\hat{V}$ with elements $V(\boldsymbol{R})_{m, m^{\prime}}, m$ denoting the magnetic sublevels of the atomic state (angular momentum $F)$ and $\boldsymbol{R}$ the atomic CM position [1]. The operator $\hat{V}$ acts on a $(2 F+1)$-component spinor wavefunction $\psi_{m}(\boldsymbol{R})$, $m=-F, \ldots, F$. The off-diagonal terms $V(\hat{\boldsymbol{R}})_{m, m^{\prime}}, m \neq$ $m^{\prime}$, describe magnetic-dipole couplings induced by a static $\boldsymbol{B}$-field, and/or stimulated Raman transitions of the atoms between different $m$-states, induced by the interaction of the atoms with two different polarization components of the lattice laser field.

Considering $\boldsymbol{R}$ a classical parameter of $\hat{V}$, the eigenenergies and eigenstates of $\hat{V}$, defined by $\hat{V}\left|\alpha_{n}(\boldsymbol{R})\right\rangle=$ $E_{n}(\boldsymbol{R})\left|\alpha_{n}(\boldsymbol{R})\right\rangle, n=1, \ldots, 2 F+1$, represent the periodic adiabatic potentials $E_{n}(R)$ of the optical lattice and the associated adiabatic eigenstates $\left|\alpha_{n}(\boldsymbol{R})\right\rangle=\sum_{m} c_{n, m}(\boldsymbol{R})|m\rangle$. The full Hamiltonian $\hat{H}=\left(\hat{\boldsymbol{P}}^{2} / 2 M\right)+\hat{V}$ can be represented in the basis of the $\left|\alpha_{n}(\boldsymbol{R})\right\rangle$ (adiabatic basis). In the adiabatic basis $\hat{H}$ contains kinetic terms that result from the action of $-\left(\hbar^{2} / 2 M\right) \nabla_{R}^{2}$ on the position-dependent coefficients $c_{n, m}(\boldsymbol{R})$. In certain optical lattices, the CM motion of the atoms is much slower than the internal spin dynamics, i.e. the spin wavefunction adiabatically follows the CM motion. In such cases, the kinetic terms of the Hamiltonian that are offdiagonal in the $\left|\alpha_{n}(\boldsymbol{R})\right\rangle$ representation are negligible. The BOA amounts to dropping those terms, thereby decoupling the Schrödinger equation for a $2 F+1$-component spinor wavefunction into $2 F+1$ scalar Schrödinger equations. If the off-diagonal kinetic terms cannot be neglected, the BOA does not apply - the motion is not adiabatically separable. Lattices that do not satisfy the BOA include the 'bright' optical lattices [1] that are red-detuned from an $F \leftrightarrow F+1$ transition, and the far-off-resonant lattice discussed in section 6 .

In our one-dimensional grey optical lattices the lowest adiabatic potential $E_{1}(z)$ is well separated from all other adiabatic potentials. Further, it is known and we observe in our quantum Monte Carlo wavefunction (QMCWF) simulations [13] that laser cooling accumulates the atoms on
$E_{1}(z)$. Thus, we are only interested in an accurate description of the dynamics on the isolated adiabatic potential $E_{1}(z)$, for which we can make the BOA. While we drop the offdiagonal kinetic terms of $\hat{H}$ in the $\left|\alpha_{n}(\boldsymbol{R})\right\rangle$ representation, the diagonal terms need to be kept, including a positive scalar potential [10],

$$
G_{n}(z)=-\left(\hbar^{2} / 2 M\right)\left\langle\alpha_{n}(z)\left|\partial_{z}^{2}\right| \alpha_{n}(z)\right\rangle .
$$

In contrast to the usual Born-Oppenheimer adiabatic potentials $E_{n}(z)$, which are proportional to the lattice intensity, the gauge potentials $G_{n}(z)$ do not depend on the lattice intensity. Since the atoms move on the lowest potential, the potential the atoms experience is the sum $E(z)=G_{1}(z)+E_{1}(z)$. Since the gauge potential involves the second derivative of the adiabatic state $\left|\alpha_{1}(z)\right\rangle$, its relative importance is very sensitive on the length scale over which $\left|\alpha_{1}(z)\right\rangle$ varies. In many lattices and other physical systems it turns out that $E_{1}(z) \gg G_{1}(z)$ for all $z$, in which case the gauge potential can be dropped.

\section{Experimental procedure}

The measurement of the tunnelling current described in the following is real-time and nondestructive, as it uses tunnelling-induced coherent photon exchange and does not introduce spontaneous emission that would destroy the coherence of the tunnelling oscillation.

In our cycled experiment [7], ${ }^{87} \mathrm{Rb}$ atoms are collected in a magneto-optic trap, pre-cooled in an optical molasses, and then loaded into a $1 \mathrm{D}$ grey optical lattice. The lattice is blue-detuned by $\delta=6 \Gamma /(2 \pi)(\Gamma /(2 \pi)=6 \mathrm{MHz})$ from the $F=2 \leftrightarrow F^{\prime}=2$ hyperfine (HFS) transition of the D1 line $(\lambda=795 \mathrm{~nm})$. The lattices are formed by linearly polarized laser beams with orthogonal (lin $\perp$ lin) or arbitrary (lin $\theta$ lin) angles between the polarization directions. After $\sim 1 \mathrm{~ms}$ of Sisyphus cooling in the lattice, most atoms are prepared in the minima of the lowest adiabatic potential $E_{1}(z)$. The wells of $E_{1}(z)$ are located at positions where the lattice light field has pure $\sigma^{+}$- or $\sigma^{-}$-polarization; at those locations the light-shift $E_{1}(z)$ is zero. Since from well to well the light polarization alternates between $\sigma^{+}$and $\sigma^{-}$, there are two types of wells, which we call $\sigma^{+}$and $\sigma^{-}$wells. The spacing between neighbouring wells of the same kind is $\lambda / 2$. Atoms located in the $\sigma^{+}\left(\sigma^{-}\right)$wells are predominantly in the $|m=2\rangle$ $(|m=-2\rangle)$ internal state.

To initiate observable tunnelling between the $\sigma^{+}$and $\sigma^{-}$wells of the lattice, all atoms located in one type of well need to be removed. We can, for instance, implement an approximation to the projection operator $|m=2\rangle\langle m=$ $\left.2\left|\int_{z}\right| z\right\rangle\langle z| \mathrm{d} z$, which will mostly remove atoms from the $\sigma^{-}$ wells. Experimentally, this is done by the application of an auxiliary $\sigma^{+}$-polarized laser pulse that is resonant on the $F=2 \leftrightarrow F^{\prime}=2$ HFS transition of the D2 line. This projection pulse, which is $\sim 15 \mu \mathrm{s}$ long, optically pumps most atoms in the $\sigma^{-}$lattice wells into the inert $5 S_{1 / 2} F=1$ state, while it leaves the atoms in the $\sigma^{+}$wells largely untouched. The end of the pulse marks the beginning of the tunnelling oscillations. To prevent the removed atoms from returning into the lattice, the repumping laser, which is needed for the 
laser cooling of the atoms, is turned off shortly before the projection pulse is turned on.

Since ${ }^{87} \mathrm{Rb}$ atoms in neighbouring wells have opposite values of $m$ with $|m|=2$, a tunnelling event is associated with an exchange of $4 \hbar$ angular momentum between the atom and the lattice field, amounting to an exchange of two photon pairs between the $\sigma^{+}$and $\sigma^{-}$components of the lattice beams. Thus, utilizing the fact that all $\sim 10^{6}$ atoms in the lattice tunnel are in phase, we can measure the tunnelling current, $0.25 n(t) \frac{\mathrm{d}}{\mathrm{d} t}\langle\hat{m}\rangle(t)$, by separating the lattice beams after their interaction with the atoms into their $\sigma$-polarized components, and measuring the difference of the intensities of the $\sigma^{+}$and $\sigma^{-}$components. As the atoms tunnel back and forth, thereby producing an AC tunnelling current, the number $n(t)$ of atoms in the $F=2$ ground state decreases, since the lattice light slowly optically pumps the $F=2$ population into the $F=1$ ground state. Since atoms in the $F=1$ state are inert, the decrease of $n(t)$ results in an overall decay of the tunnelling current. This reduction has been properly considered in the simulations of the experiment shown in figure 5.

\section{Intensity dependence of the tunnelling frequency}

For the lin $\perp$ lin lattice described in section 3 we have measured how the tunnelling frequency depends on the lattice beam intensity, and we have found that the experimentally observed tunnelling frequency quantitatively equals the maximal separation between the lowest pair of bands (see figure 1 and [7]). In figure 1 we present a theoretical analysis that allows us to understand the observed dependence. In curve $(a)$, the tunnelling frequency, defined as the maximal separation of the lowest pair of bands, is plotted versus the lattice intensity using exact band structure calculations. This curve agrees with our measurements in [7]. We compare the exact band separations to band separations obtained with a number of models. In curve $(b)$, the band structure is calculated for a scalar potential that equals the lowest lightshift potential plus its gauge potential, $E_{1}(z)+G_{1}(z)$. This model fits the exact model well, except in the range of very low intensities. In $(c)$, the same calculation is repeated for the adiabatic light-shift potential $E_{1}(z)$ only. Curve $(c)$ still fits the exact model reasonably well. Nevertheless, the inclusion of the gauge potential significantly improves the BOA-based model, particularly in the intensity range around $10 \mathrm{~mW} \mathrm{~cm}^{-2}$.

The physical reality of the gauge potential can be seen best in cases where, due to the absence of a relevant $F^{\prime}=$ $F-1$ level, there is no light-shift at all, i.e. the adiabatic potential $E_{1}(z)$ is identical to zero and $G_{1}(z)$ is the only potential that can possibly trap atoms and induce tunnelling. Curve $(d)$ in figure 1 is obtained from an exact band structure calculation for such a case. The dotted line $(e)$ shows the band splitting of the corresponding adiabatic model, i.e. a scalar particle moving on the intensity-independent gauge potential $G_{1}(z)$. At high enough intensities, where the BOA becomes valid, curves $(d)$ and $(e)$ agree pretty well. We conclude that there exist cases where the BOA is valid, and the gauge potential as the only nonzero potential governs the particle dynamics.

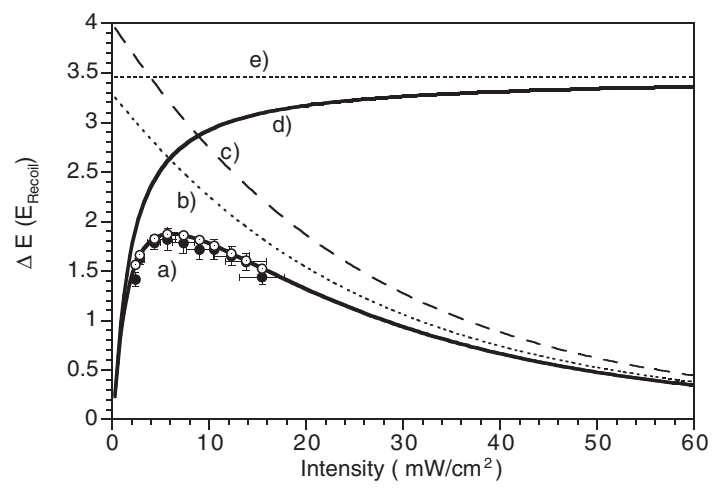

Figure 1. Maximum band splitting between the lowest pair of bands in units of the recoil energy, $E_{\text {Recoil }}=h^{2} /\left(2 \lambda^{2} M\right)$, versus the single-beam laser intensity in a lin $\perp$ lin optical lattice of ${ }^{87} \mathrm{Rb}$ on the D1 line. The detuning with respect to the $F=2 \leftrightarrow F^{\prime}=2$ HFS transition is $+6 \Gamma$. The curves are obtained from band structure calculations for the following models: (a) exact band structure model, $(b)$ adiabatic model for a scalar potential $E(z)=E_{1}(z)+G_{1}(z),(c)$ adiabatic model for $E(z)=E_{1}(z)$ only, (d) exact band structure model with the $F^{\prime}=1 \mathrm{HFS}$ level in the excited state removed, $(e)$ adiabatic model for $(d)$ with $E(z)=G_{1}(z)$. The data points show the flopping frequencies obtained experimentally $(\bullet)$ and by QMCWF simulations $(\odot)$.

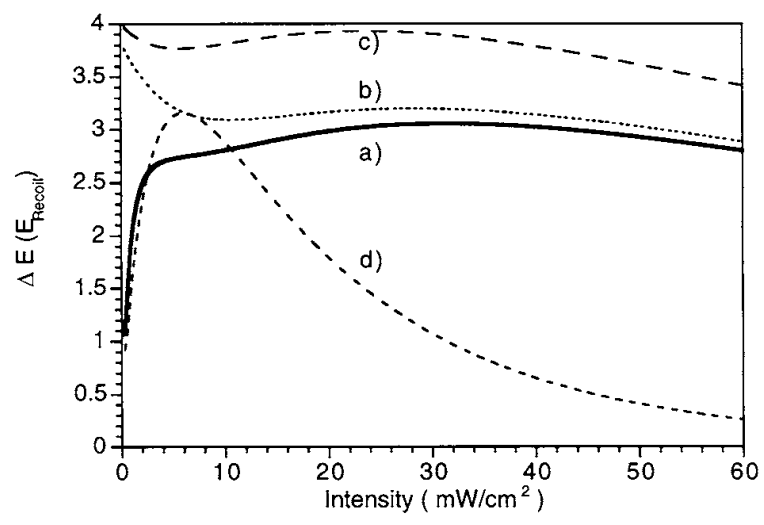

Figure 2. Maximum band splitting between the lowest pair of bands versus the single-beam laser intensity in a lin $\theta$ lin optical lattice on the D1 line of a model atom that is identical to ${ }^{87} \mathrm{Rb}$, except that the nuclear spin is $\frac{1}{2}$ instead of $\frac{3}{2}$. The laser detuning is $+6 \Gamma, \theta=1.0 \mathrm{rad}$, and the $F^{\prime}=0$ to $F^{\prime}=1$ HFS splitting in the excited state is $135 \Gamma$. The underlying assumptions are: $(a)$ exact band structure model, $(b)$ adiabatic model for a scalar potential $E(z)=E_{1}(z)+G_{1}(z),(c)$ aiabatic model for $E(z)=E_{1}(z)$ only, $(d)$ weak-coupling model, explained in section 6 .

Below $\sim 10 \mathrm{~mW} \mathrm{~cm}^{-2}$ the curve $(a)$ in figure 1 diverges from curves $(b)$ and $(c)$, and curve $(d)$ diverges from $(e)$. Both curves $(a)$ and $(d)$ go to small band separation, in accordance with the increase of the tunnelling period at low intensities we have measured in [7]. The curves $(b),(c)$ and $(e)$, which reflect tunnelling frequencies expected on the basis of the BOA, do not show the slightest indication of such a trend. We can thus conclude that the low-intensity decrease of the tunnelling frequency is caused by the breakdown of the BOA, as already suspected in [7] and further supported in the following.

In cases where the BOA does not apply, the atomic dynamics can be dominated by the motion on the diabatic potentials, $V_{m, m}(z)=\langle m|\hat{V}(z)| m\rangle$. The nondiagonal terms 

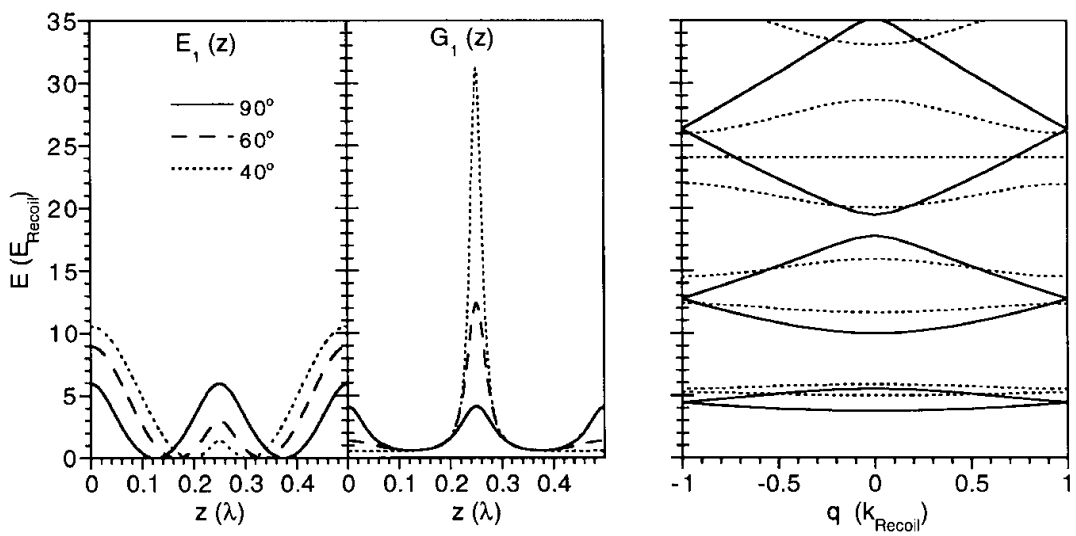

Figure 3. Lowest adiabatic potentials (left), associated gauge potentials (middle) and band structures (right) of a lin $\theta$ lin optical lattice of ${ }^{87} \mathrm{Rb}$ on the $\mathrm{D} 1$ line with the indicated values of $\theta$, a detuning of $+6 \Gamma$ with respect to the $F=2 \leftrightarrow F^{\prime}=2$ HFS transition, and $I=10 \mathrm{~mW} \mathrm{~cm}^{-2}$.

of $\hat{V}$ in the $|m, z\rangle$ representation cause couplings between the quantum states supported by the diabatic potentials. If these couplings are weak enough, the observed flopping frequency may follow from a weak coupling model $\dagger$ explained in section 6. In figure 2 we compare band splittings obtained with the weak-coupling model to the results of corresponding exact calculations and adiabatic models. For simplicity, the comparison is carried out for a grey optical lattice using a model atom with an $F=1 \leftrightarrow F^{\prime}=1$ transition. The figure shows that the adiabatic models are appropriate at higher intensities, whereby the consideration of the gauge potential again provides a significant improvement. The weakcoupling approximation is mostly bad, but it qualitatively reproduces the drop in the band splitting observed at the very low intensities. We thus conclude that, as we reduce the laser intensity, we tune the system from a range where the BOA applies into a regime where the weak-coupling approximation applies.

A qualitative understanding of why at very low intensities the band separation goes to zero can be obtained as follows. In our grey optical lattices, the weak-coupling approximation applies in the limit of very low intensity, where plane waves with fixed $m,\langle z \mid \psi\rangle=\exp (\mathrm{i} k z) \otimes|m\rangle$, are almost eigenstates of the lattice. The lattice beams weakly shift and couple those states by driving recoil-induced resonances [14] between them. The band structures of the low-intensity grey lattices are similar to that of a free particle, each free-particle band being split up by the weak atom-field interaction into a set of $2 F+1$ close-by bands. As the lattice intensity approaches zero, the atom-field interaction into a set of goes to zero, and each set of $2 F+1$ bands converges into the corresponding free-particle band. This implies that for $F \geqslant 1 / 2$ the maximum splitting between the lowest two bands goes to zero.

$\dagger$ We note that one may take the point of view that what we call the weakcoupling approximation is also a BOA, with the spin variable being the slow and the $\mathrm{CM}$ variable the fast degree of freedom. However, to avoid confusion and to be in line with common applications of the BOA, we use the term $\mathrm{BOA}$ exclusively for the case where the internal degree of freedom evolves much faster than the CM degree of freedom.

\section{Dependence of the tunnelling frequency on the polarization angle}

For the $\operatorname{lin} \theta$ lin grey lattice described in section 3 , we have studied the tunnelling current as a function of the angle $\theta$. Figure 3 displays the adiabatic potentials $E_{1}(z)$, the corresponding gauge potentials $G_{1}(z)$ and the band structure for three values of $\theta$. The figure shows that, as the angle is reduced from $90^{\circ}$, the height of the tunnelling barrier in the lowest adiabatic potential becomes smaller, while the depth of the gauge potential increases by a large amount. Thus, it is not entirely unexpected that the tunnelling rates decrease with $\theta$, as can be concluded from the separations between the lowest pairs of bands (figure 3 , right panel). This behaviour, which is the opposite to what one would conclude based on the adiabatic potential $E_{1}(z)$ alone, has been observed experimentally and in QMCWF simulations.

Figure 4 shows experimental data of the tunnelling current for different values of $\theta$ and two intensities. Our simulations, shown in figure 5 , are in good agreement with the experiment. As $\theta$ is reduced or increased from $90^{\circ}$, the tunnelling frequency reduces in a symmetric fashion, i.e. the frequency only depends on $\left|\theta-90^{\circ}\right|$, as expected. As $\theta$ approaches $30^{\circ}$ or $150^{\circ}$, the efficiency of Sisyphus cooling, which prepares the atoms in the wells prior to the tunnelling oscillation, decreases. This has two effects. Firstly, a higher-frequency tunnelling oscillation that is associated with the population of atoms in the second pair of lattice bands becomes more prominent at small and large values of $\theta$. Secondly, the population in the lowest pair of bands gets smaller, and, as a result, the tunnelling current signal due to the fundamental tunnelling oscillation weakens. The latter effect is enhanced by the decrease of the tunnelling frequency that occurs when $\left|\theta-90^{\circ}\right|$ is increased: the tunnelling current is proportional to not only the oscillation amplitude of $\langle\hat{m}\rangle$, but also to the tunnelling frequency itself. This becomes apparent in figure 5, where at the large values of $\left|\theta-90^{\circ}\right|$ the plots of $\langle\hat{m}\rangle$ show a pronounced but slow fundamental tunnelling oscillation, whereas the tunnelling current hardly shows any signal at the fundamental tunnelling frequency.

What is the physical mechanism that explains the $\theta$ dependence of the tunnelling rate? As in section 4 , there 

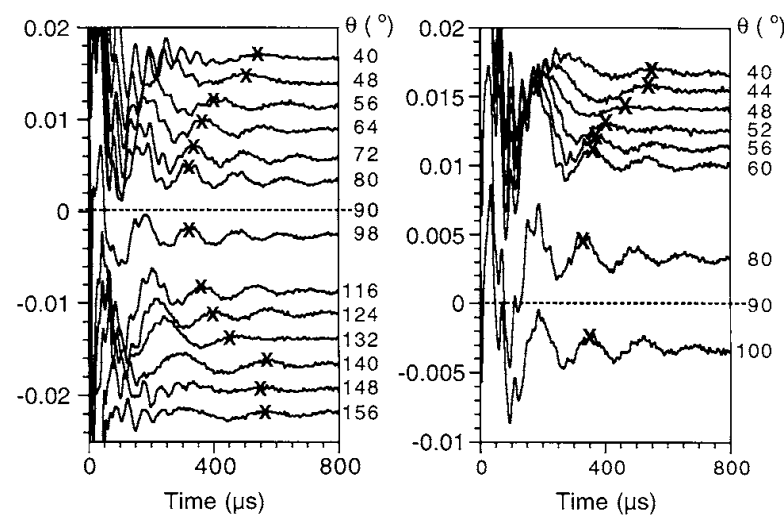

Figure 4. Experimentally observed tunnelling currents versus time in a lin $\theta$ lin optical lattice of ${ }^{87} \mathrm{Rb}$ on the D1 line with the indicated values of $\theta$ and with a detuning of $+6 \Gamma$ with respect to the $F=2 \leftrightarrow F^{\prime}=2$ HFS transition. The left panel corresponds to a single-beam intensity of $7.2 \mathrm{~mW} \mathrm{~cm} \mathrm{~cm}^{-2}$, the right one to $12.3 \mathrm{~mW} \mathrm{~cm}^{-2}$. The crosses highlight the third maxima of the curves. For clarity, the curves are offset from each other.

are two questions of interest: is the BOA valid, and what is the effect of the gauge potential? In figure 6 we show the splitting of the lowest pair of bands that corresponds to the tunnelling frequency observed experimentally and in the QMCWF simulations, for various models. Curves (a) are based on exact band structure calculations. For both intensities, the experimental and simulated oscillation frequencies $(\bullet, \odot$ respectively) taken from figures 4 and 5 agree well with curves $(a)$. Apparently, the experimental data carry a systematic error of about $5^{\circ}$ in the polarization angle. Curves $(a)$ are qualitatively reproduced by curves $(b)$, which are based on the band structures of the adiabatic model with $E(z)=E_{1}(z)+G_{1}(z)$. As expected, the qualitative agreement between is better for the higher intensity value. Curves $(a)-(c)$ and $(e)$ demonstrate that both the adiabatic and the gauge potentials need to be taken into account to achieve qualitative agreement between the exact and the adiabatic models. Curves $(d)$ and $(e)$ show that in cases where the lowest adiabatic state has zero light-shift, i.e. $E_{1}(z) \equiv 0$, the band splitting of the full spinor system is qualitatively reproduced by the band structure of the gauge potential $G_{1}(z)$. The adiabatic models are less accurate at small and large values of $\theta$, as expected from the fact that there the higher-lying adiabatic potentials start to approach the lowest one (not shown). This trend is adverse to the validity of the BOA.

We have investigated the influence of the angular momentum $F$ on the tunnelling, when all other parameters of the 'model atom' are kept constant. Generally, a larger $F$ quickly destroys the approximate validity of the BOA. This happens because, as $F$ increases, additional adiabatic potentials fill up the energy range closely above the lowest adiabatic potential, and because the order of the couplings between the internal states $|m= \pm F\rangle$ increases with $F$. The adiabatic model works best for an $F=1 \leftrightarrow F^{\prime}=1$ transition. To show this and to also make a quantitative comparison with the weak-coupling model used in sections 4 and 6 , in figure 7 an $F=1 \leftrightarrow F^{\prime}=1$ model atom is considered. The figure shows that the adiabatic models that
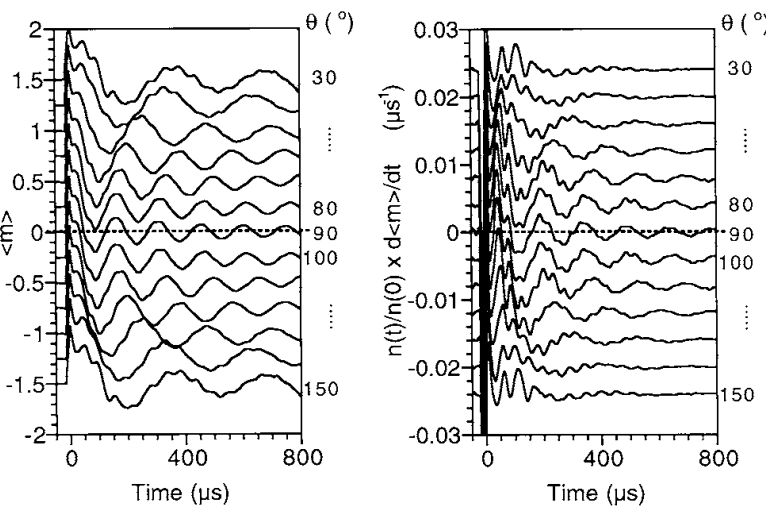

Figure 5. Simulated expectation values of $\langle\hat{m}\rangle$ and of tunnelling currents versus time for the lattice in figure 4 . The single-beam lattice intensity is $7.5 \mathrm{~mW} \mathrm{~cm}^{-2}$, and the polarization angles $\theta$ are as indicated. The number of atoms contributing to the signal is denoted $n(t)$. For clarity, the curves are offset from each other.
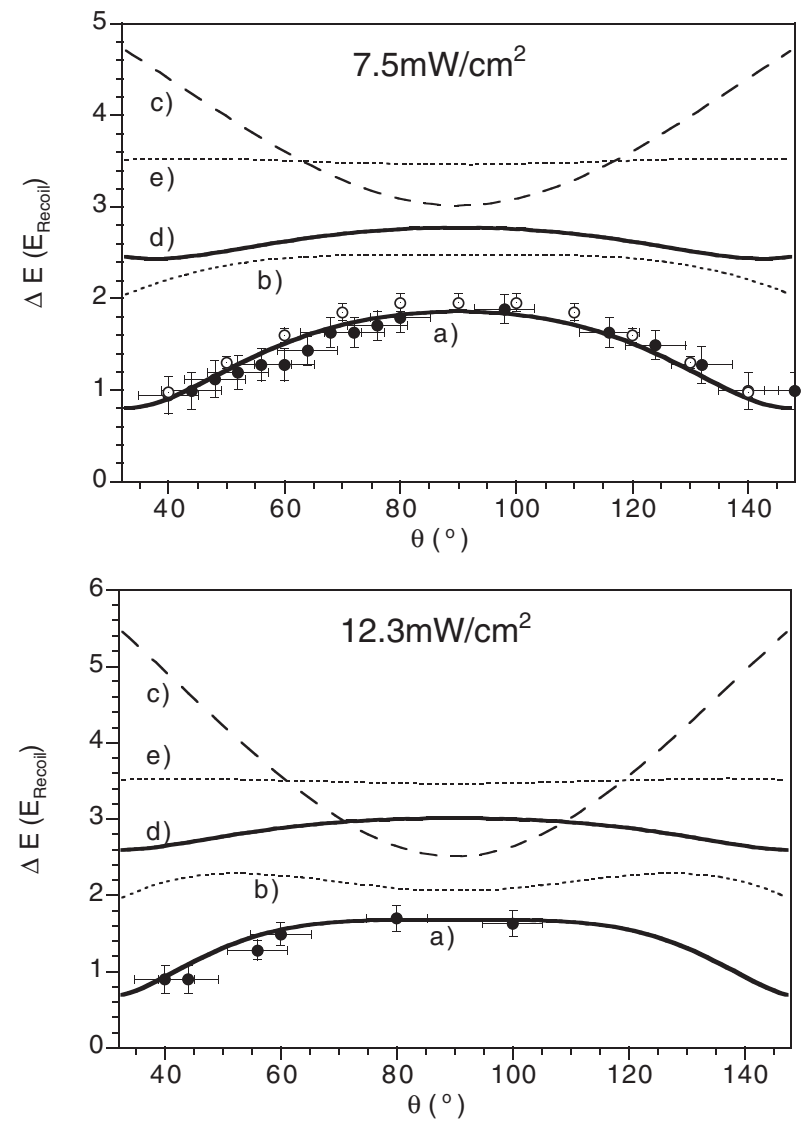

Figure 6. Maximum band splittings between the lowest pair of bands in a $\operatorname{lin} \theta \operatorname{lin}$ optical lattice versus $\theta$ for conditions as in figure 4 . The underlying assumptions are $(a)$ exact band structure model, $(b)$ adiabatic model for $E(z)=E_{1}(z)+G_{1}(z)$, (c) adiabatic model for $E(z)=E_{1}(z)$ only, $(d)$ exact model for zero light shift $\left(F^{\prime}=1\right.$ excited HFS level set very far off-resonance), $(e)$ adiabatic model for $(d)$ with $E(z)=G_{1}(z)$.

$(\bullet)$ Experimental values, mostly deduced from figure 4. $(\odot)$ values deduced from the QMCWF shown in figure 5.

include the gauge potential approximate the behaviour of the full spinor system better than in the $F=2$ case (see figure 6), and that the weak-coupling model clearly fails. 


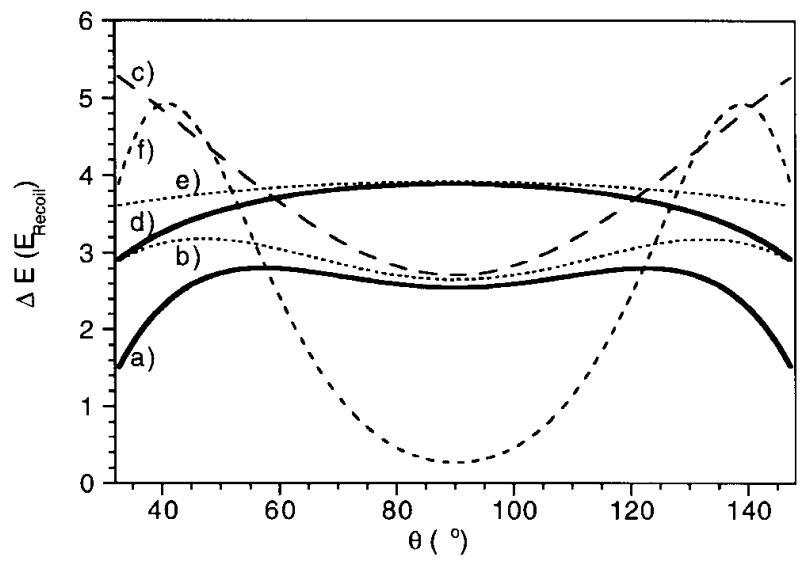

Figure 7. Maximum band splitting between the lowest pair of bands in a lin $\theta$ lin optical lattice versus $\theta$ for the D1 line of the same model atom as in figure 2 . The intensity is $I=10 \mathrm{~mW} \mathrm{~cm}{ }^{-2}$ and laser detuning $+6 \Gamma$. The underlying assumptions are $(a)$ exact band structure model, $(b)$ adiabatic model for

$E(z)=E_{1}(z)+G_{1}(z),(c)$ adiabatic model for $E(z)=E_{1}(z)$ only, $(d)$ exact model for zero light shift $\left(F^{\prime}=0\right.$ excited HFS level set very far off-resonance), $(e)$ adiabatic model for $(d)$ with

$E(z)=G_{1}(z),(f)$ weak-coupling model of the lattice used for $(a)$.

\section{Coherent well-to-well oscillations in FORLs}

At first glance, the situation we have studied in section 5 resembles the case of a far-off-resonant optical lattice in $\operatorname{lin} \theta$ lin configuration. Such lattices have been studied in [11] and also display a double well on the lowest adiabatic potential, resembling the double wells shown in figure 3. The question arises, how far the results we have obtained may apply to those lattices. We have studied the adiabatic model and a weak-coupling model for far-off-resonant lin $\theta$ lin optical lattices, and conclude that the BOA is a very bad model for this FORL, while the weak-coupling model works reasonably well.

The upper panel of figure 8 shows the lowest adiabatic potential of a far-off-resonant lin $\theta$ lin optical lattice for an $F=1 \leftrightarrow F^{\prime}=0,1,2$ model transition that is identical to the ${ }^{87} \mathrm{Rb}$ D2 transition with its HFS splittings, except that the nuclear spin is $\frac{1}{2}$, and not $\frac{3}{2}$. The single-beam intensity is $I=400 \mathrm{~mW} \mathrm{~cm}^{-2}$, the detuning with respect to the $F=1 \leftrightarrow F^{\prime}=1$ transition is $\delta_{11}=-200 \Gamma$, and $\theta=103^{\circ}$. The figure also shows the spatial probability distributions of two quantum states that are localized in only one of the two wells. These two states are not eigenstates of the system, because in their overlap region they are coupled via stimulated Raman transitions.

Two models corresponding to strong and to weak coupling can be readily tested. In the case of strong enough coupling, the above-discussed BOA can be applied, and the dynamics become effectively restricted to the lowest adiabatic potential. In that case, the fast spin degree of freedom adiabatically follows the CM dynamics, and the atoms, described by a scalar wavefunction, periodically tunnel on the potential $E_{1}(z)+G_{1}(z)$. In sections 4 and 5 this picture has been found largely appropriate.
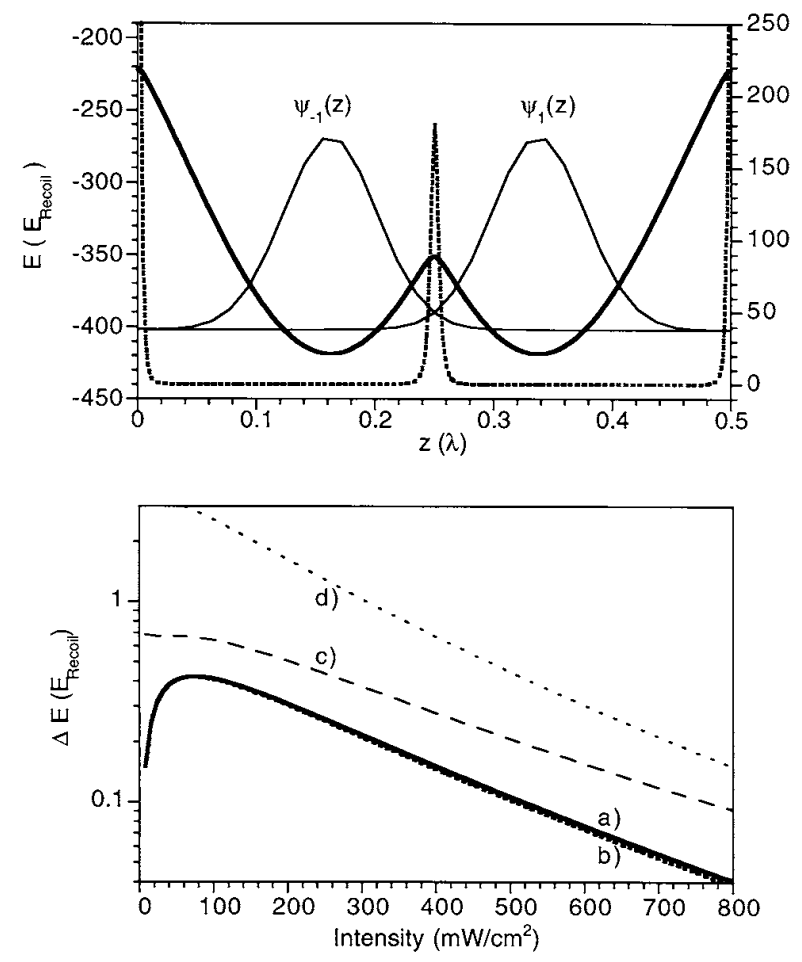

Figure 8. Upper panel: lowest adiabatic potential $E_{1}(z)$ (solid, thick, left scale), gauge potential $G_{1}(z)$ (dotted, right scale) and ground state wavefunctions in the two potential wells (solid, thin) for a $\operatorname{lin} \theta$ lin FORL of the model atom described in the text. Lower panel: maximum band splitting $\Delta E$ between the lowest pair of bands versus the lattice intensity for $(a)$ exact band structure model, $(b)$ weak-coupling model, $(c)$ adiabatic model for $E(z)=E_{1}(z)+G_{1}(z),(d)$ adiabatic model for $E(z)=E_{1}(z)$ only. Note the logarithmic scale for $\Delta E$.

In the case of weak-enough coupling, the coupling terms $V_{-1,1}(z)$ and $V_{1,-1}(z)$ in the Hamiltonian

$$
H=\left(\begin{array}{cc}
p^{2} / 2 M+V_{-1,-1}(z) & V_{-1,1}(z) \\
V_{1,-1}(z) & p^{2} / 2 M+V_{1,1}(z)
\end{array}\right)
$$

are small, and the system approximately separates into two sets of wavefunctions associated with the diabatic [1] potentials $V_{1,1}(z)$ and $V_{-1,-1}(z)$. The Hamiltonian restricted to the subspace of the lowest pair of quantum states of the diabatic potentials, which are the states shown in figure 8 , reads as

$$
H_{r}(q)=\left(\begin{array}{cc}
E_{0}(q) & c(q) \\
c^{*}(q) & E_{0}(q)
\end{array}\right) .
$$

There, $q$ is the quasimomentum, which, in cases where the weak-coupling model applies, does not have a large significance and is therefore dropped from now. The coupling $c$ in terms of the ground states $\psi_{-1}(z)$ and $\psi_{1}(z)$ is

$$
c=\int \psi_{-1}^{*}(z) V_{-1,1}(z) \psi_{1}(z) \mathrm{d} z .
$$

The energy difference between the eigenvalues of $H_{r}$ is $2|c|$. Thus, the weak-coupling model predicts a coherent oscillation of the trapped atoms between $\psi_{-1}(z)$ and $\psi_{1}(z)$ at the frequency $2|c|$.

For the above-discussed FORL, we have calculated the coupling frequencies using exact band structure calculations, 
the BOA-based model, and the weak-coupling model. The results, displayed in the lower panel of figure 8, clearly show that in the FORL the weak-coupling model fits much better than the BOA model. This conclusion is opposite to what we found in sections 4 and 5 for grey lattices. The failure of the BOA means that the well-to-well flopping in the FORL cannot be expressed in a picture equivalent to a scalar particle tunnelling on a potential surface. Nevertheless, the form of the weak-coupling Hamiltonian, equation (3), shows that in the validity range of the weak-coupling model the well-towell flopping can still be understood as a Rabi oscillation.

An increase of the $F$ quantum number will reduce the coupling strength between the degenerate $\mathrm{CM}$ quantum states associated with the diabatic potentials $V_{-F,-F}(z)$ and $V_{F, F}(z)$, because the couplings between the internal magnetic states $|m= \pm F\rangle$ will be of higher order and weaker. Thus, a larger $F$ will generally shift the picture we have obtained for the FORL even more in favour of the weak-coupling model.

\section{Conclusions and discussion}

We have used two approximations - the BOA and a weakcoupling approximation - to model the coherent well-to-well flopping of atoms we have observed in grey optical lattices. We have compared our results with coherent well-to-well flopping in a FORL. While the well-to-well flopping in our grey lattices can mostly be well described using the BOA; in the FORL the weak-coupling approximation applies.

The existence of different types of optical lattices with qualitatively different quantum transport behaviour can already be guessed from the appearance of the respective sets of adiabatic potentials. Considering the two representative cases depicted in figure 9, one is tempted to believe that the Born-Oppenheimer (weak-coupling) approximation applies in cases where the ratio between the separation $W$ of the relevant adiabatic potentials and the depth $U_{0}$ of the adiabatic potential on which the atoms mostly move is large (small). Such a comparison would, however, not reproduce the fact that for $M \rightarrow \infty$ and a fixed CM energy of order $U_{0}$ the behaviour always has to become adiabatic. A reasonable criterion can be obtained by comparing the harmonic $\mathrm{CM}$ oscillation frequency in the wells with $W$ : the BOA would then be valid if $W \gg \alpha \hbar \sqrt{U_{0} / M} / \lambda$, with a numerical factor $\alpha \approx 10$ (depending on the lattice geometry). However, this condition is questionable because of the anharmonicity of the potential wells (see figure 9), the sometimes low number of localized quantum states in the wells, and the fact that the recoil frequency $2 \pi v_{\text {Recoil }}=$ $\hbar k^{2} /(2 M)$ sets a natural scale for CM coherence frequencies. It appears reasonable to also consider the Landau-Zener crossing criterion, according to which the crossing behaviour is adiabatic if the atomic velocity $v=\mathrm{d} z / \mathrm{d} t$ at the crossing fulfils $v \ll W^{2} /\left(\hbar \Delta E_{D}(z) / \mathrm{d} z\right)$, where $\Delta E_{D}(z)$ is the position-dependent energy difference between the relevant diabatic potentials (dotted in figure 9). However, it is not clear what velocity to use, as the atomic wavefunction has a number of different momentum components, and the crossing may be found in the classically forbidden region of the wavefunction.

Since the mentioned criteria have some weaknesses and since they rarely predict the applicability of the BOA with
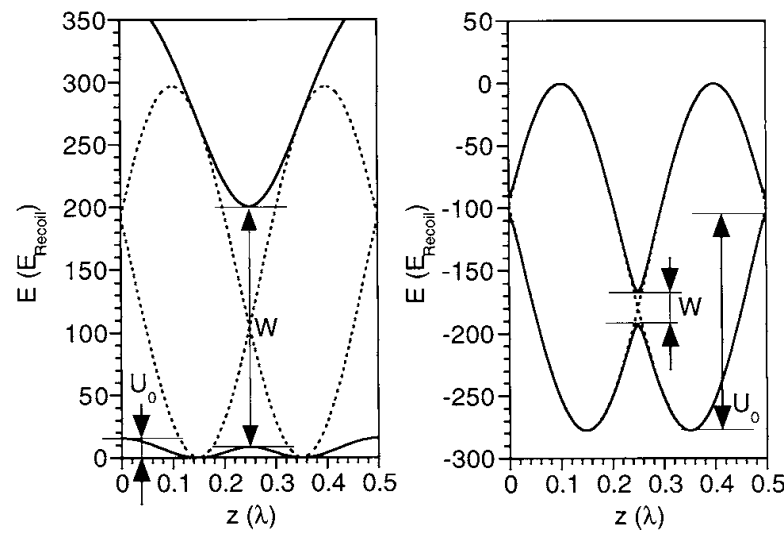

Figure 9. Left: adiabatic (solid) and diabatic (dotted) potentials of a grey optical lattice, for which the BOA is valid. Right: adiabatic and diabatic potentials of a FORL, for which the weak-coupling approximation applies. The significance of the energies $W$ and $U_{0}$ is discussed in the text.

overwhelming clarity, a detailed case-to-case analysis seems required to make qualified statements about the validity of the BOA. This is what we have done in this paper.

The signatures of coherent well-to-well oscillations in the distinct validity ranges of the BOA and of the weakcoupling approximation are the same, namely observable oscillations of the magnetic quantum number of the trapped atoms. The difference lies in the interpretation of the oscillations. It appears reasonable to call the oscillation a tunnelling phenomenon only if it can be identified with the tunnelling of a scalar wavefunction on a suitable scalar potential $E(z)$; this notion links the term 'tunnelling' with the applicability of the BOA. We have shown that the BOA applies to many grey optical lattices, with $E(z)$ being the sum of the lowest adiabatic potential, $E_{1}(z)$, and its gauge potential, $G_{1}(z)$. In the FORL which we have considered, the BOA does not apply, but the weak-coupling model does. We cannot understand the wellto-well flopping in this FORL as a tunnelling phenomenon, as we cannot map the system onto an approximately valid model that basically describes a tunnelling situation. Nevertheless, the well-to-well flopping in the FORL can still be considered as a Rabi oscillation between weakly coupled quantum states with orthogonal spin wavefunctions.

\section{References}

[1] Jessen P S and Deutsch I H 1996 Adv. At. Mol. Opt. Phys. 37 95 and references therein

[2] Haycock D L, Hamano S E, Klose G and Jessen P S 1997 Phys. Rev. A 55 R3991

Hamann S E, Haycock D L, Pax P H, Deutsch I H and Jessen P S 1998 Phys. Rev. Lett. 804149

Perrin A, Kuhn A, Bouchoule I and Salomon C 1998 Europhys. Lett. 42395

Vuletic V, Chin Cheng, Kerman A J and Chu S 1998 Phys. Rev. Lett. 815768

[3] Guo J and Berman P R 1993 Phys. Rev. A 483225

Grynberg G and Courtois J-Y 1994 Europhys. Lett. 2741

Esslinger T, Sander F, Hemmerich A, Hänsch T W, Ritsch H and Weidemüller M 1996 Opt. Lett. 21991

[4] Kozuma M, Nakagawa K, Jhe W and Ohtsu M 1996 Phys. Rev. Lett. 762428

Görlitz A, Weidemüller M, Hänsch T W and Hemmerich A 1997 Phys. Rev. Lett. 782096 
Rudy et al 1997 Phys. Rev. Lett. 784906

Raithel G, Birkl G, Phillips W D and Rolston S L 1997 Phys. Rev. Lett. 782928

Raithel G, Phillips W D and Rolston S L 1998 Phys. Rev. Lett. 813615

[5] Qian Niu, Xian-Geng Zhao, Georgakis G A and Raizen M G 1996 Phys. Rev. Lett. 764505

[6] Anderson B P and Kasevich M A 1998 Science 2821686

[7] Dutta S K, Teo B K and Raithel G 1999 Phys. Rev. Lett. 83 1934

[8] Dahan M B, Peik E, Reichel J, Castin Y and Salomon C 1996 Phys. Rev. Lett. 764508

[9] Marksteiner et al 1996 Phys. Rev. A 533409

Jurczak C, Desruelle B, Sengstock K, Courtois J-Y, Westbrook C I and Aspect A 1996 Phys. Rev. Lett. 77 1727
Katori H, Schlipf S and Walther H 1997 Phys. Rev. Lett. 79 2221

Greenwood W, Pax P and Meystre P 1997 Phys. Rev. A 56 2109

[10] Dum R and Olshanni M 1996 Phys. Rev. Lett. 761788

[11] Deutsch I H and Jessen P S 1998 Phys. Rev. A 571972

[12] Berry M V 1984 Proc. R. Soc. A 39245

Berry M V 1989 Geometric Phases in Physics ed A Shapere (Singapore: World Scientific)

[13] Dalibard J, Castin Y and Mølmer K 1992 Phys. Rev. Lett. 68 580

Marte P, Dum R, Taieb R and Zoller P 1993 Phys. Rev. A 47 1378

[14] Guo J, Berman P R, Dubetsky B and Grynberg G 1992 Phys. Rev. A 461426 